\title{
TV/Series
}

11 | 2017

Philosopher avec Battlestar Galactica

\section{Caprica, l'utopisme technologique et le « cyborg spirituel »}

\section{Mehdi Achouche}

\section{(2) OpenEdition}

\section{Journals}

Édition électronique

URL : http://journals.openedition.org/tvseries/2042

DOI : 10.4000/tvseries.2042

ISSN : 2266-0909

\section{Éditeur}

GRIC - Groupe de recherche Identités et Cultures

Référence électronique

Mehdi Achouche, "Caprica, l'utopisme technologique et le « cyborg spirituel » », TV/Series [En ligne], 11 | 2017, mis en ligne le 11 juin 2017, consulté le 01 mai 2019. URL : http://journals.openedition.org/ tvseries/2042 ; DOI : 10.4000/tvseries.2042

Ce document a été généré automatiquement le 1 mai 2019.

\section{(c) (i) (9) $\Theta$}

TV/Series est mis à disposition selon les termes de la licence Creative Commons Attribution - Pas d'Utilisation Commerciale - Pas de Modification 4.0 International. 


\title{
Caprica, l'utopisme technologique et le « cyborg spirituel »
}

\author{
Mehdi Achouche
}

What am I? The data? The process that generates

it? The relationships between the numbers? All of

the above?

(Greg Egan, Permutation City, Londres, Orion, 1994,

1 La série Battlestar Galactica (Syfy, 2003-2009) mettait en scène ce qu'Isaac Asimov appelait le « complexe de Frankenstein ${ }^{1}$ ", en référence à l'œuvre de Mary Shelley et à R.U.R. de Karel Capek (1920) : des créatures humanoïdes, en l'occurrence les Cylons, se rebellaient contre leurs créateurs et cherchaient à prendre la place de l'humanité au nom de l'évolutionnisme darwinien et de la compétition entre espèces. Les Cylons détruisaient la plus grande partie de l'humanité, la série décrivant le combat des 50000 survivants pour leur survie. Caprica (Syfy, 2010), spin-off et prequel de Battlestar composée d'une saison de 18 épisodes, si elle se situe dans le passé de son ainée, est plus originale dans sa représentation de notre rapport à la technologie, car elle imagine non pas une machine qui devient humaine ou s'humanise, mais un humain - l'humanité toute entière même -, qui devient progressivement machine. Il s'agit donc bien d'authentiques "cyborgs » tel que les avaient imaginés Manfred Clynes et Nathan Kline en 1960 : non pas un androïde cybernétique passant pour un être humain, mais un être humain "amélioré " ou « augmenté », voire radicalement transformé, par la technologie ${ }^{2}$.

Ce changement de perspective, ainsi que le fait que la série abandonne le space opera pour se situer dans une société urbaine futuriste mais très proche esthétiquement et thématiquement de la nôtre (les créateurs de la série parlent même de " soap opera ${ }^{3}$ » et de «family drama ${ }^{4}$ » futuriste), autorise Caprica à se concentrer sur ses personnages et leur relation à la technologie de façon plus soutenue et plus fine que son aînée ${ }^{5}$. Son statut de prequel lui permet de se définir comme une série pré-apocalyptique, et comme la chronique de la «techno-déchéance » d'une humanité que l'on sait déjà condamnée moins par les machines qu'elle est sur le point de créer, que par ses propres rêves de 
perfectionnement. Cette idéologie de la perfectibilité technologique, dont rarement une série télévisée se sera autant fait l'écho, prend également un tour plus inattendu en se concentrant sur des êtres humains capables de transférer ou de copier leur conscience dans des machines ou dans la réalité virtuelle - dans Galactica, seuls les Cylons en étaient capables -, rejoignant ainsi un rêve et un projet éminemment contemporains: l'immortalité digitale. La série met ainsi en évidence, pour le problématiser, le rêve de la dématérialisation de l'individu, de l'abandon du corps au seul bénéfice d'un esprit libéré de toute finitude. Elle se concentre ainsi sur les aspirations «spirituelles » qui soustendent des techno-utopies parfois moins matérialistes et rationnelles qu'elles ne le paraissent.

3 La série s'ouvre par la représentation de la lente décadence qui affecte l'humanité spéculaire du fait de la réalité virtuelle "libératrice», s'appuyant à la fois sur une rhétorique d'ordre judéo-chrétienne, sur les topoï de la science-fiction contemporaine et sur la philosophie transhumaniste pour dramatiser cette corruption endogène. Mais Caprica pousse plus loin la mécanisation et l'autonomisation de la conscience, avec la genèse d'une technologie permettant la copie numérique d'une conscience humaine, et donc l'immortalité digitale, mettant ainsi en scène une nouvelle conception de l'intelligence artificielle. Enfin, à la décadence nihiliste exposée dans la diégèse s'oppose, grâce à cette apothéose d'un genre nouveau, un projet porté par une acception spirituelle, voire religieuse, de la dématérialisation. La série met ainsi en scène la conception mécaniste du corps et le «cyborg spirituel» au cœur des utopismes technologiques actuels.

\section{La décadence virtuelle}

4 Galactica s'était conclue par la révélation que la série se situait dans notre lointain passé. La dernière scène, située à Times Square, New York, en 2009, s'achevait sur le constat de la « décadence » de notre propre société : "mercantilisme, décadence, une technologie déchaînée, ça te rappelle quelque chose $?^{6} »$. La question était suivie par des images d'archive de robots humanoïdes contemporains et les progrès fulgurants de la robotique, plans qui concluaient la série. La Terre du début $d u \mathrm{xxI}^{\mathrm{e}}$ siècle était ainsi engagée sur le même chemin suicidaire qui avait conduit Caprica et les onze autres colonies à leur perte, des milliers d'années auparavant, le cycle historique se reproduisant sans cesse. C'est ce même progrès et ce même mercantilisme délétère que Caprica choisit de mettre en scène, en remontant le temps du récit Galactica, et en décrivant le moment critique du cycle qui voit l'émergence de la première intelligence artificielle (IA), 58 ans avant la «fin du monde » qui ouvre Battlestar Galactica - fin du monde qui en est la conséquence directe.

"Caprica » désigne avant tout la polis métonymique dans laquelle se déroule l'histoire Caprica City, la cité du futur dont l'esthétique connote à la fois l'utopie futuriste traditionnelle (les gratte-ciels immenses et majestueux souvent montrés dans les plans de situation ou en arrière-plan, les aéronefs traversant le ciel à basse altitude, la beauté fuselée de l'ensemble, qui invitent tous à l'arrêt sur image et à la contemplation de ce sublime technologique) et un futur très proche du présent des spectateurs, dans la mesure où la plupart des technologies présentes à l'écran, les voitures ou les vêtements, sont similaires aux nôtres ${ }^{7}$. La série se situe ainsi « sur une planète ressemblant à la Terre, dans un avenir proche, traitant de nombre des dilemmes éthiques auxquels nous allons très bientôt devoir nous confronter ${ }^{8}$ ». La dimension allégorique de la série met en scène, 
d'après le co-créateur et showrunner de la série Ron Moore, une «société qui court à sa perte, une lueur démente dans le regard ", l'utopie apparente nourrissant en son sein les germes du désastre à venir' ${ }^{9}$. L'histoire de la série est donc l'Histoire de cette société spéculaire.

Fig. 1 : L'utopie urbaine du futur

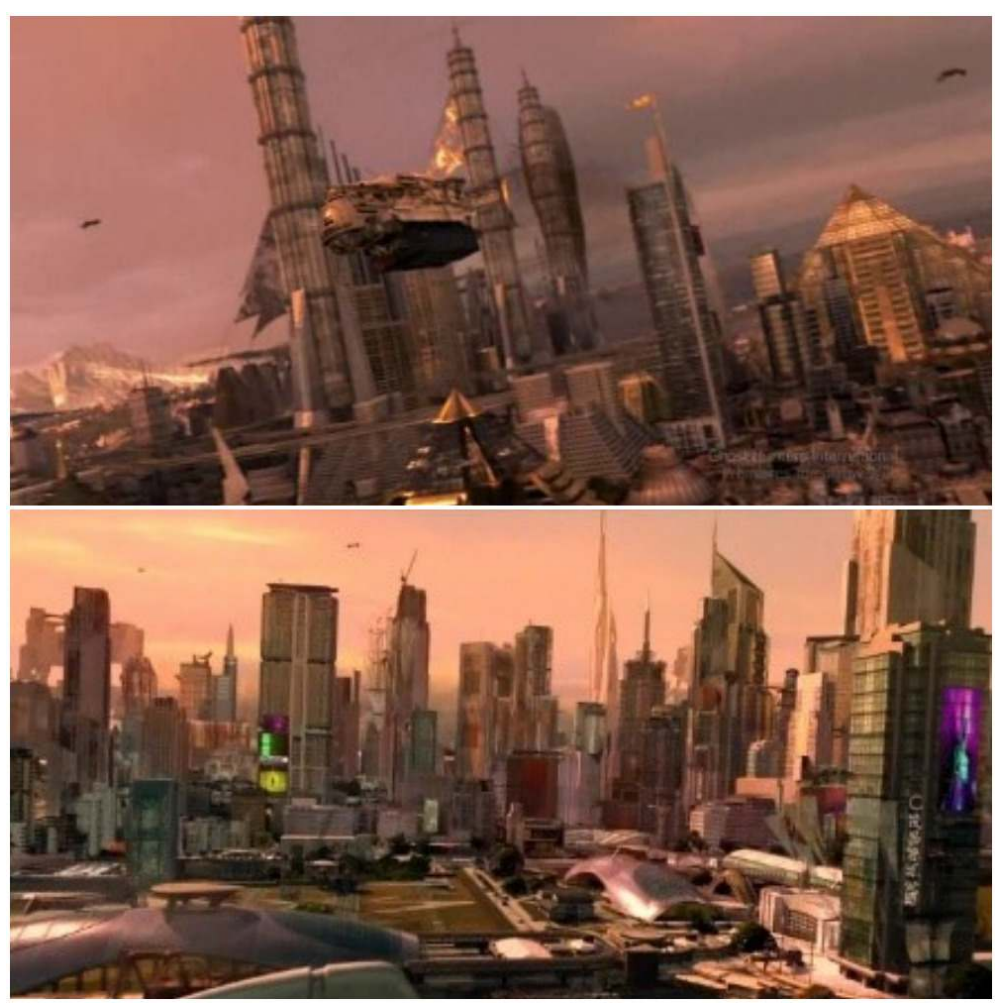

Cette lueur démente dans les yeux des citoyens de Caprica, c'est celle de l'enivrement provoqué par les progrès technologiques et scientifiques, que la série prend soin d'associer à l'hubris et au mercantilisme capitaliste. L'un des principaux protagonistes, Daniel Graystone, est à la fois un inventeur génial, qui autrefois bricolait dans le garage de ses parents, et l'un des principaux capitaines d'industrie de la planète - un technologiste semblable à un Bill Gates ou un Steve Jobs. Graystone a créé et mis sur le marché le holoband, appareil qui permet à son utilisateur de se plonger dans la réalité virtuelle, "V-World», et d'y accomplir, sous la forme d'un avatar (ici une version virtuelle similaire ou "améliorée » de l'individu d'origine), ses moindres désirs et fantasmes, très souvent à caractère sexuel ou violent. "V-World» est une sorte de littéralisation des mondes virtuels sur Internet, dont un grand nombre revêt un caractère pornographique, et, on le découvrira au cours de la série, des jeux vidéo du type « survival » ou « first-person shooter ». Mais V-World est avant tout un «metavers », un univers virtuel persistant qui dépasse le seul cadre du jeu ludique, reproduit les interactions économiques et sociales de la réalité (monnaie virtuelle, magasins en tous genres, etc.) et n'obéit pas à un scénario particulier, tout en instaurant ses propres règles et lois ${ }^{10}$. Or, la règle ici est qu'il n'existe justement pas de règles - « pas de limites, c'est la devise $^{11} »$. La scène d'ouverture du pilote nous montre, par l'entremise d'un montage rapide qui accentue l'impression de chaos, une boite de nuit, "V-Club ", à l'ambiance gothique, où se déroulent dans la pénombre, au son d'une musique trance comme le montrent de rapides vignettes, des orgies, des combats ultra-violents ou même des 
meurtres gratuits acclamés par une foule en délire, le clou du spectacle étant l'exécution d'une vierge sur scène, encadrée par des danseuses lascives aux masques bestiaux et démoniaques.

7 La liberté qu'est censée promettre la technologie se transforme donc en licence, les jeunes gens se livrant à un hédonisme nihiliste, s'amusant à braver les interdits et passant le plus clair de leur temps dans un monde virtuel où tout est permis, puisque rien n'a de conséquence dans le monde réel. Ils profitent donc paradoxalement de leur désincarnation pour jouir sans entrave de leurs corps et du plaisir des sens. Des scènes ultérieures montreront un jeu de roulette russe ("There Is Another Sky », S01E05) ou un gang de jeunes voyous habillés et armés à la façon du gang d'Orange Mécanique (Stanley Kubrick, 1971) ${ }^{12}$, connotant encore davantage la nature jouissive, anarchique et ultraviolente du V-World (« Unvanquished », S01E10). C'est pourquoi le holoband et le V-World, sources de la colossale fortune de Graystone, sont la cible de critiques d'ordre moral, alors que ce qui préoccupe Graystone est surtout sa marge bénéficiaire ("Gravedancing ", S01E05). Le divertissement capitaliste, les jeux vidéo et une réalité virtuelle enfin photoréaliste et totalement immersive sont donc synonymes d'échappatoire malsain et de disparition des cadres éthiques et moraux traditionnels - thème classique du cinéma de science-fiction contemporain ${ }^{13}$.

8 La série s'organise ainsi autour de trois niveaux de réalité et de trois temporalités: Caprica City (1), le reflet partiellement futuriste de la société des téléspectateurs (2), et « New Caprica City » (3), la fiction dans la fiction. "New Cap City » est le reflet virtuel exact (chaque rue, bâtiment et appartement y a son alter ego digital) et dystopique de Caprica City, où l'on peut observer les comportements qui menacent la cité dans son propre avenir. (En effet, on peut imaginer que les comportements visualisés dans « New Caprica City » finiront par se reproduire dans le monde réel, ce qui sera confirmé par le dernier épisode.) Le Nouveau Monde utopique ou dystopique n'est donc plus une autre planète, comme dans le space opera en général et dans Galactica en particulier, mais celui créé par la technologie virtuelle, qui devient l'enjeu de luttes entre les différents camps qui s'affrontent dans Caprica City pour l'investir de leur propre sens. La série abandonne ainsi le thème de l'illusion et du simulacre, populaire dans les années 1990 (Matrix), pour se concentrer sur la contamination morale du réel par le virtuel. 
Fig. 2 : Le holoband et la réalité virtuelle omniprésente



Tout comme Galactica, Caprica et son paratexte publicitaire utilisent la rhétorique et l'imaginaire judéo-chrétien pour dramatiser la « décadence » morale à l'œuvre, procédant dans un premier temps à une opposition classique entre technologisme et religiosité. L'humanité est sur le point de croquer la pomme du savoir (cf. fig. 3) - ou l'a déjà croquée - et va donc être expulsée du jardin, alors que, fort logiquement, le dénouement de Battlestar Galactica consiste en l'abandon de toute technologie au profit du jardin d'Éden qu'était la Terre préhistorique. Galactica faisait constamment référence à «La Chute » pour qualifier la destruction de l'humanité par ses machines, terme présent dans l'incipit de Caprica: la scène de la discothèque infernale illustre cette chute. Cette dernière désigne dès lors moins la destruction de l'humanité par la menace extérieure que sont les machines $^{14}$ que la lente désintégration de la société dont la ville Caprica est la synecdoque - une chute au ralenti, une lente décadence, finalement punie par Dieu. Les trois jeunes gens mis en scène dans le «V-Club » dès les premières minutes du pilote et qui ne goûtent pas le spectacle de cette débauche ("Ils ne savent pas ce qu'ils font », dit l'un d'eux, citant les paroles christiques ${ }^{15}$ ) se réfugient dans une église ou chapelle virtuelle, synonyme à leurs yeux de valeurs plus saines qu'ils vont chercher à promouvoir dans la réalité virtuelle comme dans le monde réel, lançant ainsi l'arc narratif principal de la série (des fondamentalistes religieux cherchent à réformer le monde, ce qui conduit rapidement à la mort de deux des trois jeunes gens aperçus). La série mobilise donc une rhétorique de la chute dans son paratexte mais met également celle-ci en abyme à travers sa diégèse et ses personnages, jouant des ironies de ce discours. 
Fig. 3 : Images promotionnelles qui soulignent la nature de la tentation à laquelle sont soumis les personnages

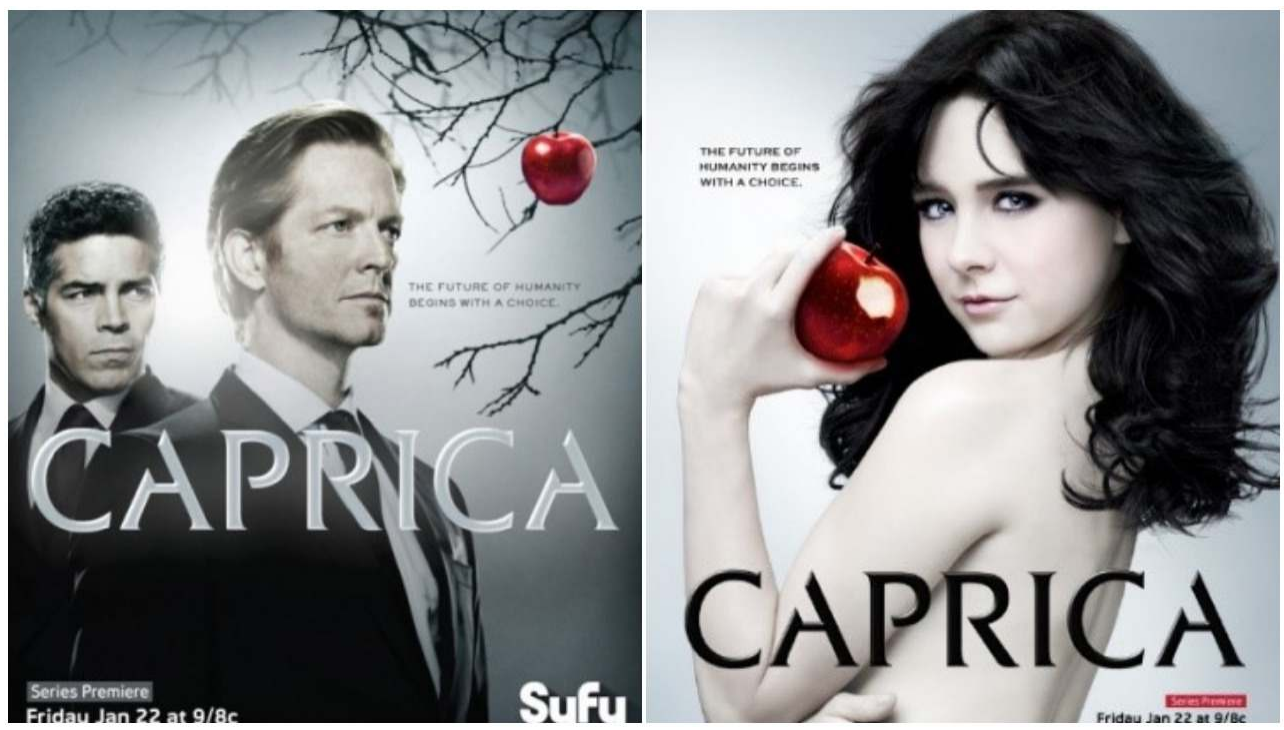

10 S'il représente une variation sur le thème du "savant fou », Graystone, dont la tour géante ouvre le générique et trône sur la ville, incarne avant tout un "capitaliste fou » typique du cinéma de science-fiction contemporain. Le personnage est très proche des idéaux transhumanistes actuels, et de ces techno-utopistes ultra-matérialistes (dont plus d'un est à la fois un technologiste et un homme d'affaires) dont on verra que l'imaginaire est directement confronté à l'argumentaire religieux. Les transhumanistes rêvent de créer une Intelligence Artificielle, immensément plus intelligente que l'être humain, qui pourra donc accélérer le progrès humain de façon soudaine et irréversible, transformant irrévocablement le monde - un événement le plus souvent appelé la "Singularité ». L'autre grand rêve transhumaniste, rendu possible par l'IA, consiste à vaincre la mort, notamment en «copiant » et téléchargeant la conscience humaine sur un disque dur, dans une redéfinition du corps humain en termes de « hardware » et de la conscience et de l'esprit en termes de «software ». L'idée hante la science-fiction depuis les années 1980 et l'avènement de l'ordinateur personnel et d'Internet, le courant cyberpunk souscrivant souvent à la même dualité cartésienne :

Un robot, ou une personne, est composé de deux parties : le matériel et le logiciel [...]. Votre cerveau est le matériel, tandis que l'information contenue dans le cerveau est le logiciel. L'esprit [...] les souvenirs, les habitudes, les opinions, les savoir-faire [...] relèvent tous du logiciel ${ }^{16}$.

Hardware et software humains constituent donc le wetware, la machine humaine - l'homme est bien en définitive une machine, et l'a toujours été, comme l'écrivait La Mettrie en 1748 ${ }^{17}$. Et si l'homme, à l'instar de l'animal dans la pensée de Descartes, peut être considéré comme une machine, alors il s'ensuit logiquement, pour le transhumanisme, que l'on pourra intervenir sur le corps humain (et animal) pour y réaliser toutes les transformations souhaitées, car si l'homme est une machine, on peut donc le mécaniser encore davantage. Dans le célèbre roman Neuromancer (1984), un pirate informatique désormais incapable de se connecter au « cyberespace » a connu « la Chute. [...] Le corps était de la viande. Case était tombé dans la prison de sa propre chair ${ }^{18} "$, renversement 
ironique de la Chute biblique et rapport à la « chair » correspondant à celui présenté dans Caprica.

Fig. 4 : Le dualisme corps/esprit comme enjeu sociétal majeur à Caprica

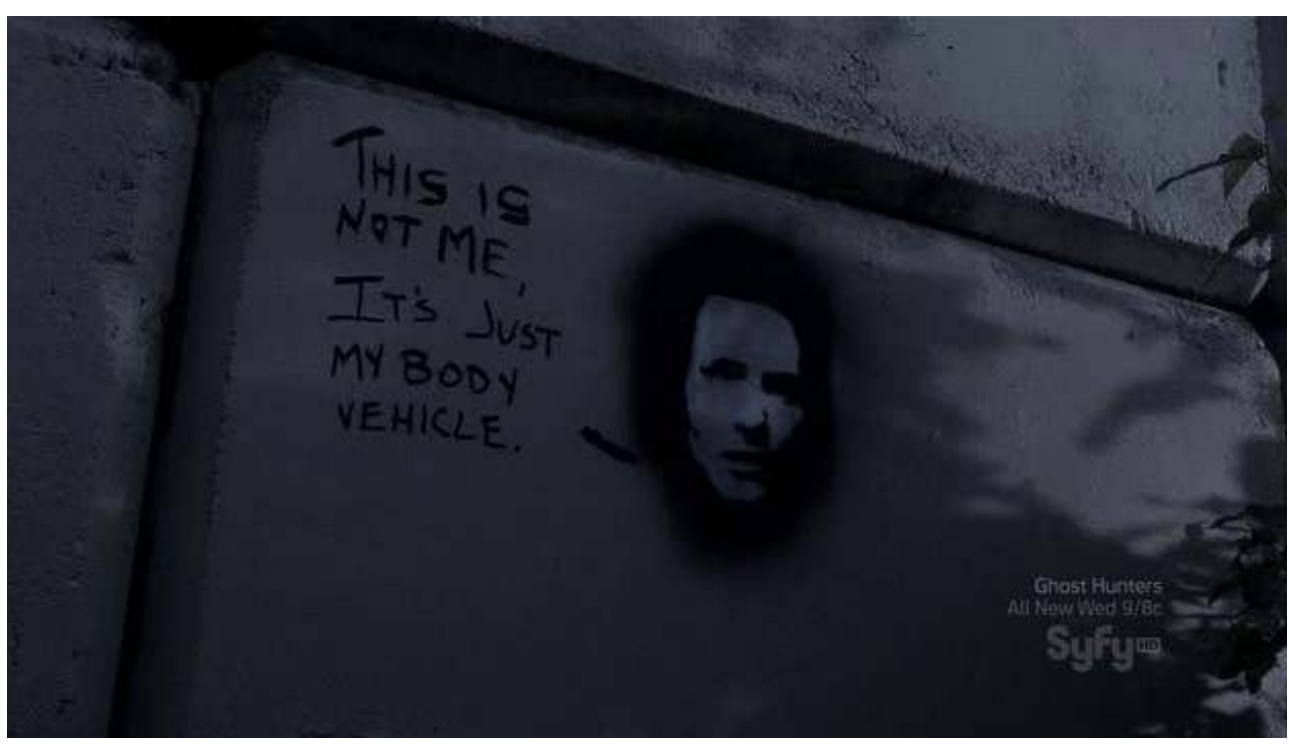

Pour les transhumanistes il n'existe pas de limites au progrès, puisque le corps humain lui-même représente la nouvelle "frontière ", pouvant être modifié ou "amélioré ", donnant naissance au posthumain. En cela ils souscrivent à la célèbre réflexion de Pic de la Mirandole :

\begin{abstract}
Si nous ne t'avons fait ni céleste ni terrestre, ni mortel ni immortel, c'est afin que, doté pour ainsi dire du pouvoir arbitral et honorifique de te modeler et de te façonner toi-même, tu te donnes la forme qui aurait eu ta préférence. Tu pourras dégénérer en formes inférieures, qui sont bestiales; tu pourras, par décision de ton esprit, te régénérer en formes supérieures, qui sont divines ${ }^{19}$.
\end{abstract}

Le transhumanisme se voit ainsi comme le digne héritier de l'humanisme historique, continuant le travail de l'évolution naturelle, ce que Michel Serres appelle le " xénodarwinisme ${ }^{20}$ ", en considérant l'être humain comme un être en devenir, un « work in progress ». Le transhumanisme accepte et encourage donc toutes les modifications et les trans-formations - «l'ère du trans » est en marche, jusqu'à atteindre les formes « divines » promises par la Mirandole. Comme l'a écrit un autre célèbre transhumaniste, Marvin Minsky, les robots sont bien notre avenir... mais nous serons les robots en question ${ }^{21}$.

14 Caprica met donc en scène une décadence provoquée par la technologie. Contrairement au scénario de la série mère Galactica, les Cylons passent rapidement au second plan pour céder le devant de la scène au V-World et aux entités désincarnées qui le peuplent. La série met en scène une technologie plus contemporaine encore de la nôtre, ainsi qu'une nouvelle forme d'intelligence artificielle qui, dans la continuité des thèses transhumanistes, valide jusqu'à un certain point la dualité corps/esprit. Par ailleurs, si la religion et la spiritualité sont d'abord convoquées pour condamner ce progrès, Caprica entreprend rapidement de brouiller la donne morale de son récit. 


\section{Les fantômes dans la machine}

15 La fille de Graystone, Zoé, est tuée dans un attentat terroriste durant l'épisode pilote. Mais, en technologiste géniale, elle avait auparavant créé un avatar à son image, "Zoé$\mathrm{A}$ ", au sein du V-World, grâce à une méthode que de nombreux transhumanistes défendent aujourd'hui : scanner et « simuler» le cerveau d'une personne (« whole brain emulation »). Des entreprises, comme Eterni.me par exemple, proposent aujourd'hui de recueillir l'empreinte digitale d'un individu - c'est-à-dire les innombrables informations qu'une personne égrène tout au long de sa vie sur Internet et les réseaux sociaux - et de transférer cette empreinte digitale dans un avatar en ligne qui « reconstruira » ensuite la personnalité de l'individu - une forme «d'immortalité digitale ${ }^{22}$ ». Martine Rothblatt, figure de proue du transhumanisme et de la sauvegarde digitale de la personnalité d'un individu, nomme les composants fondamentaux de la personnalité des "bèmes ", plus décisifs selon elle pour l'avenir de la (trans)humanité que les gènes, parce qu'ils permettraient de reconstruire la personnalité d'une personne après sa mort, comme elle l'a déjà fait pour son épouse ${ }^{23}$. «Elle [Zoé] a pris un moteur de recherche et l'a transformé en outil pour tromper la mort ! », résume un peu vite Graystone euphorique, une idée que la série Black Mirror a aussi elle aussi récemment mise en scène ${ }^{24}$.

Graystone décidera même d'exploiter l'idée à des fins mercantiles, commercialisant l'invention sous le nom de programme «Résurrection». Il décrit son idée à un investisseur potentiel de la façon suivante, paraphrasant presque le site officiel d'Eterni.me :

Un remède au deuil. [...] Qui n'a pas perdu un être cher? Qui ne ferait pas n'importe quoi pour le ramener? Et si tout ce dont vous aviez besoin pour le faire était d'acheter le bon logiciel? Ils seraient dans le monde virtuel, c'est vrai, mais vous pourriez leur rendre visite tous les jours, passer à nouveau du temps avec eux, leur dire toutes les choses que vous n'aviez pas eu le temps de leur dire. Peut-être qu'un jour nous pourrons même trouver un moyen de leur fournir un corps dans le monde réel. Ils feraient à nouveau partie de nos vies. Le médicament ultime. Pour guérir la douleur ultime. Combien cela vaut-il à votre avis ${ }^{25}$ ?

17 Graystone, dans un spot publicitaire diffusé d'abord en plein écran, comme s'il s'adressait aux spectateurs de la série, les interpelle ainsi, en regard caméra :

Imaginez ne plus jamais avoir à dire au revoir aux êtres qui vous sont chers. Imginez un futur sans deuil, disponible dès aujourd'hui. Grâce, par Graystone Technologies. Parce que certains souvenirs devraient vivre éternellement ${ }^{26}$.

Il continue donc d'exploiter les faiblesses humaines en promettant d'éradiquer la mort : il utilise cyniquement l'imaginaire chrétien ("Résurrection ", " Grâce ») et réduit le rêve à un slogan publicitaire en proposant un remède technologique "miracle». Le spot publicitaire va jusqu'à mettre en scène un militaire mort au combat qui retrouve sa famille durant ses propres obsèques (pour un maximum de pathos), tandis que Graystone et ses acolytes discutent cyniquement des manières de toucher encore davantage le public familial. La série souligne ainsi, par cette mise en abyme de la télévision, le caractère mercantile et manipulateur d'un techno-utopisme qui se résume ici à une rhétorique publicitaire hypocrite («imaginez un monde où...»). Elle renvoie enfin les spectateurs à leur propre statut de téléspectateurs, par l'interpellation «imaginez un monde..." . 
Graystone et l'autre grand protagoniste de la série, Adama (le père du futur commandant du Galactica, tout aussi sceptique que lui face au progrès technologique), abordent le sujet de l'immortalité digitale dans le pilote, sous forme de débat religieux. Graystone, qui n'a que faire des rêves de désincarnation des uns et des autres, cherche à rendre à " sa fille " une forme physique, en téléchargeant son esprit désincarné dans un corps cybernétique. Il défie donc la mort au nom de valeurs transhumanistes matérialistes et initie un débat éthique avec Adama, au prénom très biblique de Joseph (que les scénaristes prennent soin de souligner durant le débat). Ce débat auquel les téléspectateurs sont convoqués constitue une scène archétypale du cinéma de science-fiction. Aux arguments transhumanistes de l'un répondent les idées traditionnalistes de l'autre, qui s'exclame que l'idée d'un avatar intelligent et conscient fait à l'image d'une personne disparue n'est rien d'autre qu'une «abomination ». Graystone, qui dans le script du pilote lui répondait que ce n'était pas une abomination mais un " miracle ${ }^{27}$ ", a besoin des services d'Adama et fait figure de tentateur, de Méphistophélès hyper-rationaliste lorsqu'il cherche à convaincre son interlocuteur, qui a perdu sa propre fille, Tamara, lors du même attentat, de vaincre la mort. Affirmant que notre personnalité n'est qu'une somme d'informations, de données traitées par le processeur naturel qu'est le cerveau mais qui pourraient tout aussi bien être téléchargées dans un véritable processeur, il demande à Joseph horrifié, "Qui sait si son âme n'a pas, elle aussi, été copiée ? ${ }^{28}$ ", blasphème qui marque son appartenance à la famille des "savants-fous ». Il poursuit, empruntant cette fois la rhétorique transhumaniste et matérialiste : "Vous savez ce qu'est votre cerveau, Joseph ? Une base de données et un processeur, rien de plus. De l'information, et un système pour l'exploiter ${ }^{29} »$. Après tout, comme le souligne aussi l'avatar de Zoé pour expliquer son existence, «le cerveau humain contient 300 giga-octets d'informations, pas tant que ça quand on y pense $\mathrm{e}^{30}$ ». Les transhumanistes ne diraient pas mieux que le père et la fille Graystone, eux qui souscrivent littéralement à l'adage technologiste contemporain: « Tout est information ».

20 C'est donc au nom d'un tel réductionnisme que la conscience de Zoé peut être « recopiée » et « téléchargée » dans le V-World, puis une nouvelle copie de celle-ci copiée sur un disque et téléchargée dans le corps d'un robot, puis à nouveau " téléchargée » dans le V-World, et enfin, dans un processus apparemment infini, téléchargée dans un corps cybernétique humanoïde à la toute fin de la série. Comme l'explique Graystone, après tout, la peau, les yeux, les cheveux sont des " détails de surface », " ce qui compte est ce qui est à l'intérieur, comme on dit à nos enfants ", c'est-à-dire l'esprit, la conscience «stockée » dans le cerveau. Le transhumaniste Graystone rappelle ainsi implicitement Frankenstein dans sa volonté de (re)créer la vie. En " téléchargeant », contre la volonté de celle-ci, l'esprit de sa fille décédée dans le corps d'un de ses robots (ce que le script compare explicitement à la démarche de Frankenstein ${ }^{31}$ ), Graystone fait du "fantôme " de sa fille sa création et créature (celle-ci décidera d'échapper à son « arrogance » pour mener à bien ses propres projets, s'enfuyant bientôt dans la réalité virtuelle).

21 Enfin, Graystone reprend à son compte l'un des grands arguments transhumanistes dans son débat avec Adama : « définissez ce qui est naturel. Mes lunettes m'aident à voir, les membres et les organes artificiels aident des millions de gens à vivre, vous pourriez difficilement les qualifier de naturels, pourtant je doute que vous les qualifiez d' abominations». On pourrait répondre à Graystone, à la suite de nombreux critiques contemporains, que ces technologies sont supposées guérir, "réparer », le corps humain à la manière de prothèses, et non l'augmenter ou lui assurer une forme d'immortalité. 
Mais la mort n'est-elle pas, du point de vue transhumaniste, une maladie dont il faut guérir? Graystone pose ainsi une question fondamentale, invitant le spectateur à se la poser à son tour : qu'est-ce, au final, que le «naturel »? Comment peut-on définir cette notion lorsque l'on parle des êtres humains, et doit-on fatalement en appeler à l'argument religieux pour le définir? On serait tenté de paraphraser un autre «savantfou ", cette fois de cinéma: puisque Dieu ou la nature a fait l'homme, tout ce que fait l'homme est donc voulu par Dieu, et est donc " naturel ${ }^{32}$ ». En posant cette question, la série commence donc dès le pilote, par l'entremise de Graystone, à compliquer le débat en déstabilisant la binarité essentialiste séparant naturel et artificiel.

Durant le même débat, Graystone souligne que Zoé-A «est une copie mais... c'est une copie parfaite ${ }^{33}$ ». Pour lui, la réalité et la "virtualité $»^{34}$ ne peuvent ontologiquement s'opposer puisque, comme le veut selon Graystone la devise qui prévaut dans le secteur de la réalité virtuelle du futur, « une différence dont on ne perçoit pas la différence n'est pas une différence », citation intradiégétique du philosophe William James ${ }^{35}$. La réflexion recoupe en partie le célèbre test de Turing, pour qui une intelligence artificielle pourrait vraiment être considérée comme intelligente et consciente s'il était tout à fait impossible de faire la différence entre elle et un être humain. Qu'importe donc si Zoé-A est une copie artificielle, elle n'en est pas moins "intelligente» et surtout consciente, dotée des souvenirs, de la personnalité et de tout ce qui constituait sa fille, si ce n'est son corps physique. Comme la citation de James le souligne, le caractère artificiel de Zoé-A est sans importance. Mais la question même de son artificialité est interrogée: est-elle une intelligence «artificielle » créée par Zoé, ou n'est-elle pas plutôt le résultat d'un " transfert » de l'esprit de cette dernière, la reproduction à l'identique d'une intelligence « naturelle »? Si l'on devait un jour réussir à réaliser la copie d'un esprit humain, et créer ainsi une deuxième conscience identique à la première qui serait téléchargée par exemple dans une machine - un " mindclone ${ }^{36}$ " -, aurait-on vraiment affaire à une intelligence artificielle imitant un humain, ou plutôt à une nouvelle acception du cyborg, un être naturel délivré de son corps ou copié (ou simulé ?) par la technologie ${ }^{37}$ ? S'il s'agit là d'une artificialisation du vivant, peut-on vraiment qualifier un tel être d'artificiel ?

La publicité pour le programme « Résurrection », aussi cynique qu'elle soit, ne ment donc (techniquement) pas, ce qui en constitue tout le danger : on pourra réellement retrouver des êtres chers... même si les avatars de ceux-ci finiront irrémédiablement par s'éloigner de leur " être-source» pour développer leur propre personnalité38. Si Graystone refuse tout d'abord de reconnaître à l'avatar une véritable personnalité et une conscience autonome ( $u$ une personne est bien plus qu'un ensemble de données $\left.{ }^{39} »\right)$, il change rapidement d'avis et reconnaît une humanité à part entière à un personnage qui devient le protagoniste de la série - Zoé a bien copié sa conscience dans le monde virtuel, la série validant ainsi la dichotomie corps/esprit. La technique de copie d'une conscience a bien créé deux «fantômes dans la machine », comme le veut le titre d'un épisode, reprenant ainsi le célèbre titre d'un ouvrage du philosophe Gilbert Ryle ${ }^{40}$. L'ironie est que Ryle avait trouvé cette formule pour rejeter ce qu'il appelle le «mythe de Descartes » (c'est-à-dire, le dualisme cartésien), alors que des œuvres comme Caprica y souscrivent clairement (même si c'est pour critiquer, dans leur mise en abyme, l'ambition transhumaniste ${ }^{41}$ ).

De fait, l'avatar, après avoir été "scanné », développe progressivement ses propres expériences, ses propres souvenirs, donc sa propre personnalité ( Je n’ai pas l'impression de n'être qu'une copie $\left.\mathrm{e}^{42} »\right)$. La saison peut alors se lire comme la lente individualisation et humanisation de Zoé-A, passant d'écho spectral de Zoé (mystérieusement recouverte de 
sang après la mort de cette dernière, elle doutera ensuite de sa propre existence) à un individu à part entière (elle finit par comprendre sa "raison d'être » dans le dernier épisode). La série pose donc, à la suite des transhumanistes, la question de l'artificialité - où s'arrête le naturel et où commence l'artificiel (ou inversement dans le cas de Zoé-A) ? Et comment définir ces deux notions?

$\mathrm{Du}$ pilote qui met l'accent sur la décadence sociale et l'hubris transhumaniste du " capitaliste fou », la série évolue ainsi vers une représentation plus inattendue de cette nouvelle technologie en examinant la création et l'évolution de Zoé-A, progressivement caractérisée comme un être à part entière et véritable héroïne de la série. Cette dernière va encore davantage compliquer les termes du débat en représentant les religieux intradiégétiques comme les plus fervents technologistes de Caprica.

\section{L'apothéose technologique}

Si Graystone développe ses inventions à des fins purement commerciales, sa fille Zoé a des objectifs bien différents en tête. Graystone crée une simulation ludique grâce au holoband, mais Zoé parvient à créer une IA, son avatar, afin de transformer le V-World: Zoé-A représente la Singularité, qui peut apparaître comme le véritable thème de la série. Zoé, qui est aussi une hackeuse, veut notamment transformer "New Cap City ", où règnent le chaos, la violence et la criminalité, en un lieu utopique où la technologie pourra vraiment se faire libératrice. Elle crée donc Zoé-A, présentée pour la première fois dans une église (ou chapelle) qui est l'antithèse de la boîte de nuit décadente, et qui est censée purifier le V-World. Après la mort de Zoé, Zoé-A, avec l'aide de Tamara-A, veut à son tour purifier la réalité virtuelle et l'ensemble de la société. Elle représente donc une acception de la technologie et de ses promesses à l'opposé de celles de son "père ». Le soap opera dont se réclame Caprica met ainsi en scène sous forme de conflit familial l'opposition contemporaine entre utilisations de la technologie - commerciale et militaire d'un côté, subversive de l'autre.

Zoé agit aussi au nom de ses propres croyances religieuses. Caprica est une société polythéiste qui vénère le panthéon gréco-romain; certains accusent ce monde "païen " de décadence. Les orgies et les sacrifices $\mathrm{du}$ « $\mathrm{V}$-Club » sont par exemple conduits au nom d'Hécate, « déesse des Enfers ", tandis que des combats de gladiateurs ont également lieu dans le V-World (« Things We Lock Away », S01E12). Zoé, elle, croit en un Dieu unique, de même qu'un groupuscule monothéiste fondamentaliste appelé les "Soldats de l'Unique " ("Soldiers of The One»). La série renvoie donc à la naissance du christianisme sous l'Empire romain, tout en la réécrivant : cette "secte », comme la définissent les autorités, n'est pas montrée comme victime d'une répression religieuse, mais comme responsable des attentats à la bombe commis à Caprica City - dans le pilote de la série, un kamikaze se fait exploser dans le métro au nom de ce Dieu (dit «One True God»).

Or, cette secte, loin de dénoncer la technologie, les holobands et le monde virtuel du VWorld, décide au contraire de les embrasser et de les incorporer à sa foi. Le téléchargement de l'esprit («mind uploading») inventé par Zoé, ainsi que le V-World, permettront de créer un autre monde - virtuel mais existant bel et bien -, un Paradis où les «martyrs » de la foi (les kamikazes), et bientôt l'ensemble des croyants, deviendront comme des Dieux puisqu'immortels, vivant dans le confort et l'aisance éternelle. Plutôt que de s'immerger provisoirement dans un environnement virtuel, les êtres humains pourront définitivement abandonner leur corps et transférer leur esprit dans une réalité 
virtuelle qui pourra leur offrir une forme de béatitude. Le nom du programme informatique censé les amener dans cette utopie virtuelle, Apothéose ("Apotheosis »), dit bien les choses: ces chrétiens fondamentalistes veulent utiliser la technologie pour atteindre la forme "divine" que promettait La Mirandole et à laquelle rêvent les transhumanistes. L'une des leaders du groupe, Sœur Clarice, ayant démontré ce que peut cette technologie, en vante ainsi les mérites, décrivant le transfert de l'esprit des croyants vers ce paradis virtuel :

Imaginez un monde dans lequel la mort a été conquise. Dans lequel la vie éternelle n'est pas juste un rêve mais une réalité, [...], une vie éternelle dans un paradis virtuel que nous avons construit ${ }^{43}$.

« Imaginez un monde... » : Clarice retrouve les termes mêmes de Graystone pour charmer son auditoire, faisant miroiter un avenir radieux grâce à la technologie qu'elle a dérobée au scientifique. Quoiqu'antagonistes (Graystone parviendra dans le dernier épisode à contrecarrer les plans terroristes de la secte de Clarice), ils partagent le même rêve techno-utopique, dont ils se servent pareillement pour manipuler les foules. Un membre de son auditoire souligne d'ailleurs malicieusement à quel point Clarice excelle dans l'art de la rhétorique publicitaire. L'utopisme est donc à nouveau présenté comme un mode de discours "vendeur» qui peut servir les desseins les plus mercantiles ou les plus meurtriers.

Fig. 5 : Le Paradis virtuel créé par Sœur Clarice

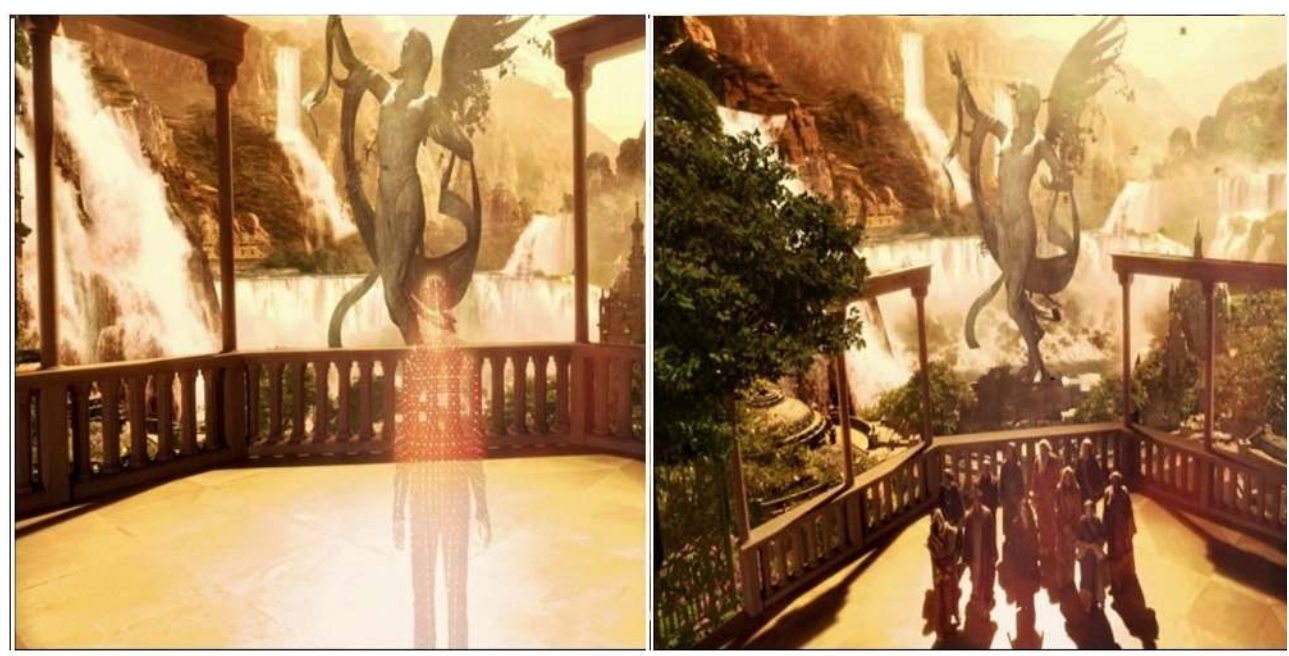

L'immortalité telle que conçue par les transhumanistes n'est pas foncièrement différente des croyances religieuses, dit en substance la série, et religion et science peuvent être réconciliées. Clarice poursuit :

Nous vivons une ère remarquable. Les mythes et les mystères ont été remplacés par la raison et la science. Je vous offre une religion qui élimine la nécessité de croire. Une religion de certitude, qui reflète le merveilleux de tout ce que nous avons créé ${ }^{44}$

31 Religion et technologie pourront donc faire bon ménage, et ce n'est pas tant à se rapprocher de Dieu que travaillent les «Soldiers of the One " qu'à transformer leurs membres en divinités, ce qui, comme l'explique le personnage, garantira le triomphe de leur religion dans les douze colonies. Merveilleux instrument de marketing religieux donc, qui prend le contre-pied des fêtes païennes orgiaques mais participe de la même entreprise que les nihilistes du V-Club ou les actionnaires de Graystone Industries : créer, 
grâce à la technologie, le monde virtuel parfait, libérateur ou hyper-rentable, anarchique ou "paradisiaque "... tout en servant les intérêts de ses initiateurs (Graystone veut s'enrichir et ramener sa fille d'entre les morts, Clarice veut des statues à son image). Le danger technologiste n'est pas donc tant qu'il pourrait mener à des conséquences inattendues (la révolte des machines) mais à des conséquences prévisibles.

L'ironie dramatique qui plane sur ce récit est l'apocalypse qui résultera de cette ambition, la Chute originelle. L'avatar de Zoé, ainsi que celui de Tamara, qui habitent désormais le V-World, découvrent progressivement leurs pouvoirs au sein de ce monde dont elles peuvent contrôler les lois physiques à l'inverse des autres utilisateurs - deux "divinités " ont bien été créées et comme le crie Zoé-A, dans le dernier épisode, « Dieu c'est moi ! $^{45}$ ». Elle finira par s'opposer au plan des "Soldiers of the One ", comprenant le risque que représente le discours utopiste :

Si les gens savent qu'ils vont aller au Paradis quoi qu'il arrive, ils vont perdre le contrôle qu'ils ont sur eux-mêmes. [...] Le monde réel va se transformer en jeu. Ça sera comme à New Cap City, les gens vont tuer, violer, détruire ${ }^{46}$.

Le rêve de l'apothéose virtuelle se transformera donc en cauchemar, les comportements humains s'alignant sur ceux que l'on peut trouver sur Internet et dans les jeux-vidéo. La divinité Zoé-A, plus fidèle au dogme chrétien que Sœur Clarice (le Paradis est supposé se mériter et non se monnayer) détruit donc ce Paradis artificiel, mais les deux fantômes dans la machine « hantent » toujours les circuits. Surtout, Zoé-A, d'instrument de Zoé, est devenue agent libre, réalisant ses propres choix éthiques. L'« intelligence artificielle » qui doutait de sa propre identité (" Rebirth », S01E02) détruit ainsi les rêves techno-utopistes de Clarice et s'oppose au projet qui est pourtant à son origine, compliquant encore un peu plus la représentation initiale.

Un discours comme celui véhiculé par la série rejoint donc les analyses qui décèlent un caractère religieux, mystique ou spirituel dans le rêve techno-utopiste contemporain. Erik Davis, dans son ouvrage TechGnosis, met en exergue le parallèle entre ce rêve et les aspirations gnostiques, c'est-à-dire un ensemble de croyances du début de l'ère chrétienne qu'on rassemble sous le terme de "gnosticisme». Pour les gnostiques, le monde physique a été créé imparfait par un démiurge maléfique ou incompétent, tel le " malin génie » cartésien, et le but est d'échapper à cette pseudo-réalité pour atteindre la connaissance et se libérer du monde matériel inférieur. Le corps est le reflet de cette réalité physique repoussante, caractérisée par la souffrance et l'ignorance, tandis que l'esprit constitue l'étincelle divine de l'individu, et son espoir de transcender la réalité quotidienne. La Chute du jardin d'Éden n'est pas le drame originel mais le premier acte de libération d'un individu qui accède à un début de connaissance et de lucidité. Davis met en parallèle ces croyances, qui n'ont jamais tout à fait disparu de l'imaginaire occidental, et les utopies technologiques comme celle des transhumanistes, soulignant les nombreux points de recoupement entre elles. C'est pourquoi il nomme «cyborg spirituel » la manifestation contemporaine de ce désir multiséculaire de s'élever au-dessus des limites du monde physique grâce à une fusion entre l'esprit et la machine ${ }^{47}$. Un film récent, Transcendence (Wally Pfister, 2014) voyait aussi son propre technologiste décider de renommer la Singularité: «Certains l'appellent la Singularité. Je préfère l'appeler 'Transcendance' ${ }^{\prime 4}$ ".

L'ironie dans la série consiste enfin à voir la "divinité » qu'est Zoé-A renoncer à son statut pour rejoindre le monde réel, dans la toute dernière scène de la série. Mais, dans une ironie dramatique, le montage-séquence de quatre minutes et demie qui clôt la série, 
intitulé "The Shape of Things to Come" (référence ambivalente au roman à la fois dystopique et utopique de H.G. Wells) et situé cinq ans années plus tard ${ }^{49}$, nous laisse apercevoir le futur proche. "Jetons un coup d'œil au futur ", propose un journaliste qui interviewe Graystone, nous ramenant une nouvelle fois à notre statut de téléspectateurs, occupés à visionner un "reportage " sur notre propre futur. Les propos de Graystone, presque en excipit de la série, sont lourds d'ironie. Il se félicite de voir ses robots de plus en plus intégrés à la société, où ils constituent une nouvelle classe servile :

Je pense que les gens sont suffisamment intelligents pour se rendre compte que, si utiles qu'ils soient, les Cylons sont juste des instruments, rien de plus, et que d'oublier cela, de brouiller la distinction entre hommes et machines, et de leur attribuer des qualités humaines, c'est de la folie ${ }^{50}$. Graystone dit cela alors que Zoé-A le regarde à la télévision, lovée dans les bras de sa mère au sein du V-World, et qu'une scène suivante le montrera en train de "ressusciter " sa fille en téléchargeant l'esprit de cette dernière dans un corps humanoïde - lui-même ne croit donc pas à ce qu'il dit. C'est en raison de leur insupportable condition servile que les Cylons, qui sont bel et bien conscients, se rebelleront contre l'humanité, comme le savent les spectateurs qui ont vu Battlestar Galactica et qui savent qu'il faudrait plutôt reconnaître aux machines leur " humanité », comme Graystone l'a déjà fait pour Zoé-A, et les traiter en conséquence, pour éviter l'apocalypse à venir. De fait, les Cylons, comme nous le montre la suite du montage, ont rejoint la foi monothéiste de Clarice, et vénèrent un Dieu qui leur reconnaît le statut d'êtres conscients et libres, eux les nouveaux esclaves de cet Empire " décadent ». Leur propre spiritualité leur fournira ainsi, comme le suggère l'épisode, l'argument pour se révolter contre leurs créateurs. Enfin, Clarice, devenue la grande prêtresse des machines, "prophétise ", durant son sermon aux robots, l'arrivée prochaine du Messie ( Je vais parler maintenant de quelqu'un qui vous apportera la liberté »), tandis que le travelling latéral sur les robots installés sur les bancs de l'église s'arrête au même moment pour faire le point sur Zoé-A qui sourit (sans que l'on sache si c'est un sourire de connivence ou un sourire ironique). 
Fig. 6 : Clarice prêchant la bonne parole aux robots

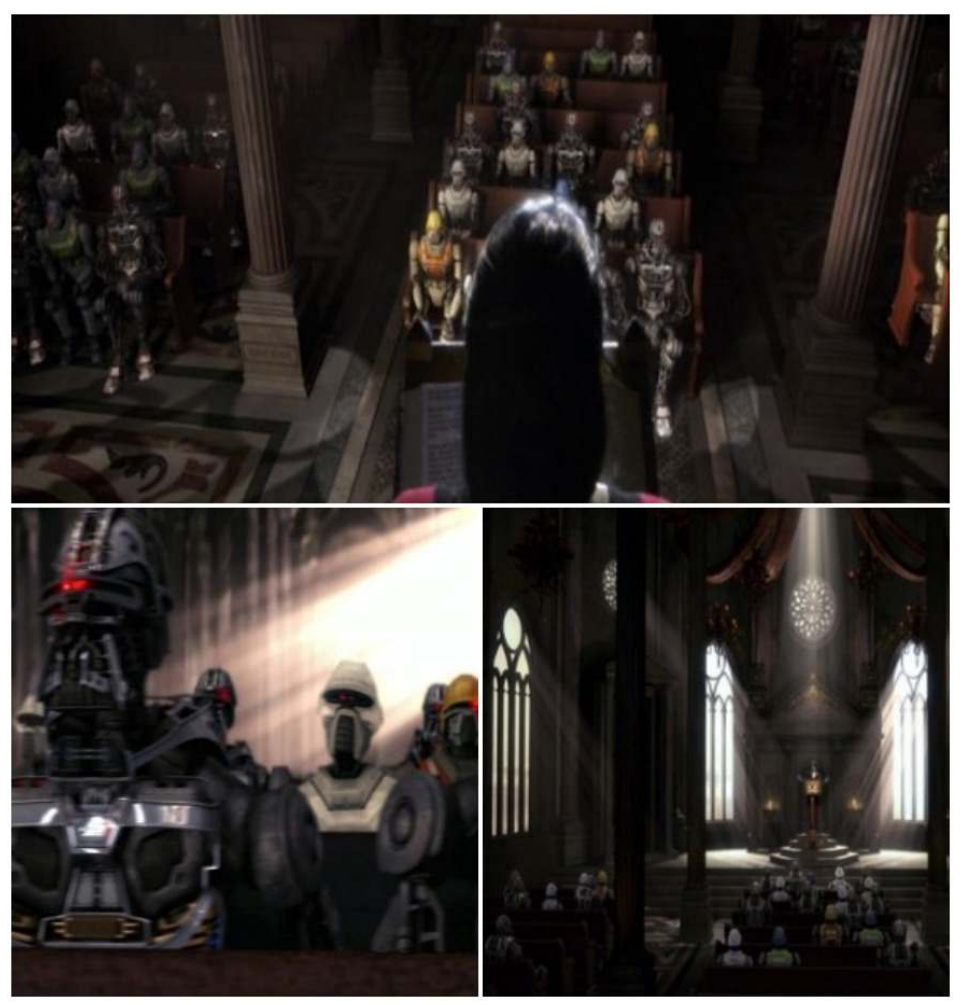

La série suggère ainsi que Zoé sera bien, malgré tout (et à travers elle, son père également), l'instrument (involontaire ?) de l'apocalypse à venir, la série se concluant par le plan de la planète Caprica qui l'avait ouverte. Dans une dernière mise en abyme, les spectateurs pourront voir dans Caprica une image de notre Terre.

\section{Conclusion}

Sans surprise, les transhumanistes se sont beaucoup intéressés à Caprica, y décelant leurs propres préoccupations ainsi qu'un traitement bienveillant de celles-ci. Plusieurs d'entre eux, comme Raymond Kurtzweil ou Martine Rothblatt, ont participé à des ateliers organisés dans le cadre de la diffusion du pilote de la série ${ }^{51}$. Certains y voient un traitement respectueux et intelligent des idées transhumanistes, puisque des intelligences « artificielles » peuvent être créées, que le dualisme corps/esprit y est validé et que les avatars Zoé-A et Tamara-A sont représentées de manière bienveillante. D'autres, au contraire, comme la revue conservatrice américaine National Review, y décèlent un avertissement contre ces mêmes idéaux transhumanistes ${ }^{52}$. Mais au-delà de l'ambiguïté de la série quant à la réalité virtuelle, l'intelligence artificielle et l'immortalité digitale, Caprica réussit surtout à s'emparer des thématiques transhumanistes pour problématiser le rapport de ces derniers, et des technologistes en général, aux technologies mélioratives. Elle souligne le caractère "spirituel » que peut revêtir le progrès technologique ou la Singularité, et illustre à quel point la dualité cartésienne peut être répandue et validée dans la culture populaire ${ }^{53}$. Annulée au bout de dix-huit épisodes, Caprica n'a pas pu continuer à explorer ces sujets et à développer son discours, mais elle a su amplifier le discours religieux de Battlestar Galactica et le déplacer vers le 
domaine de la spiritualité technologiste et transhumaniste. Elle a ainsi su problématiser une acception, à la fois très ancienne et très contemporaine, de la sublimation de l'être humain, des motivations (utopistes, mercantiles et/ou "spirituelles») qui la soustendent, et des conséquences à la fois utopiques et apocalyptiques qui en découlent. Dépassant le topos qui oppose être humain et machine, Caprica invite à une réflexion plus fine sur les motivations profondes de la philosophie transhumaniste, et montre que cette dernière, toute futuriste qu'elle soit, relève d'une histoire déjà très ancienne.

\section{BIBLIOGRAPHIE}

ACHOUCHE Mehdi, L'utopisme technologique dans la science-fiction hollywoodienne, 1982-2010 : transhumanisme, posthumanité et le rêve de «l'homme-machine », Thèse de doctorat d'études anglophones, sous la direction de Donna Spalding-Andréolle, Université Le Havre Normandie, 2011, https://hal.archives-ouvertes.fr/file/index/docid/779615/ filename/20686_ACHOUCHE_2011_archivage.pdf ASIMOV Isaac, « Robots I have Known », Robot Visions, New York, Penguin Putnam, 1991. AUBUCHON Rémi, Ronald D. MOORE, Caprica, cinquième version, 28 septembre 2006, pdf, disponible en ligne : http://leethomson.myzen.co.uk/Battlestar_Galactica/Caprica_pt1.pdf

BRADY Matt, « Sci-Fi Greenlights Caprica Series », 2 décembre 2008, http:// www.newsarama.com/1645-sci-fi-greenlights-caprica-series.html

CLYNES Manfred E., Nathan S. KLINE, « Cyborgs and Space », Astronautics, septembre 1960.

CAPEK Karel \& Joseph, R.U.R. and the Insect Play, Oxford, Oxford University Press, 1961 [1920]. CLERGEAT Romain, « Le PDG le mieux payé d'Amérique. Martine Rothblatt, transgenre et transhumaniste », Paris Match, 9 novembre 2014, www.parismatch.com/Actu/Environnement/ Martine-Rothblatt-transgenre-et-transhumaniste- 648558

DAVIS Erik, TechGnosis, New York, Harmony Books, 1998.

DOYLE C., W. Mieder, F. Shapiro, The Dictionary of Modern Proverbs, New Haven, Yale University Press, 2012, édition kindle.

DVORSKY George, « Exploring transhumanist themes in Battlestar Galactica: Caprica », Institute for Ethics and Emerging Technologies, 30 juin 2009,

http://ieet.org/index.php/IEET/more/3202/

EGAN Greg, Permutation City, Londres, Orion, 1994.

GIBSON William, Neuromancer, 1984, Londres, HarperCollins, 1995 [1984].

KEYMASTER Peter. «Mindclones from Social Media: New Research from Stanford Suggests Feasibility », H + Magazine, 15 janvier 2015, http://hplusmagazine.com/2015/01/15/mindclonessocial-media-new-research-stanford-suggests-feasibility/ 
LEWIS Tanya, « The Singularity is Near: Digital Immortality by 2045 ?», Live Science.com, 17 juin 2013, http://www.livescience.com/37499-immortality-by-2045-conference.html

MINSKY Marvin, « Will Robots Inherit the Earth? », Scientific American, octobre 1994, http:// web.media.mit.edu/ minsky/papers/sciam.inherit.html

MIRANDOLE Jean Pic de la, De la Dignité de l'homme, 1486, traduit par Yves Hersant, http:// www.lyber-eclat.net/lyber/mirandola/pictrad.html

PARKIN Simon, «Back-up brains: the era of digital immortality », BBC.com, 23 janvier 2015, http://www.bbc.com/future/story/20150122-the-secret-to-immortality

PHILLIPS Jevon, «Caprica: A Chat with Ron Moore about the sci-fi soap opera », The L.A. Times, 6 février 2010, http://latimesblogs.latimes.com/showtracker/2010/02/caprica-ron-moore.html Rотнвlatt Martine, « On Genes, Memes, Bemes, and Conscious Things », The Journal of Personal Cyberconsciousness, 1:4, 2006, http://www.terasemjournals.com/PCJournal/PC0104/ rothblatt_04a.html ROSE Lacey, « Talking TV With Syfy's Dave Howe », 12 janvier 2009, http:// www.forbes.com/2009/12/01/syfy-battlestar-galactica-business-entertainment-caprica.html ROUSSEL François-Gabriel Roussel, «Culture des métavers et culture académique », Communication [En ligne], Vol. 33/1, 2015, mis en ligne le 19 février 2015, consulté le 5 mars 2017. URL : http://communication.revues.org/5227

RUCKER Rudy. The Ware Tetralogy: Software, Prime Books, 2010 [1982], édition pdf.

RоTHмAN Peter, « Transhumanism at the Woodstock Film Festival », communiqué de presse, 26 août 2009, http://www.woodstockfilmfestival.com/press/releases/2009_08_syfy.htm RYLE Gilbert, The Concept of Mind, Londres, Routledge, 1949.

SERRES Michel, «Le temps humain : de l'évolution créatrice au créateur d'évolution », P. Picq, M. Serres, J-D. Vincent, Qu'est-ce que l'humain ?, Paris, Le Pommier, 2008.

SMITH Wesley J., « Surrogates and Caprica: Splendid Sci Fi Warnings Against Transhumanism », National Review.com, blog, 3 février 2010, http://www.nationalreview.com/humanexceptionalism/325209/surrogates-and-caprica-splendid-sci-fi-warnings-againsttranshumanism SURFDADDY Orca, « Caprica: Birth of the Cylons », H + Magazine, 19 janvier 2010, http:// hplusmagazine.com/2010/01/19/caprica-birth-cylons/

\section{NOTES}

1. Isaac Asimov, « Robots I have Known », Robot Visions, New York, Penguin Putnam, 1991, p. 409.

2. Manfred E. Clynes, Nathan S. Kline, «Cyborgs and Space», Astronautics, septembre 1960, disponible en ligne: http://web.mit.edu/digitalapollo/Documents/Chapter1/cyborgs.pdf, consulté le 10/07/2015.

3. L'idée de se rapprocher d'un soap opera était aussi censé permettre à la chaîne d'attirer un public plus large et surtout plus féminin vers l'univers Galactica, http:// latimesblogs.latimes.com/showtracker/2010/02/caprica-ron-moore.html, 6 février 2010, http:// www.forbes.com/2009/12/01/syfy-battlestar-galactica-business-entertainment-caprica.html, 12 janvier 2009, consultés le 05/08/2015. 
4. Rémi Aubuchon, "What the Frak is Caprica?", bonus DVD, Caprica, l'intégrale de la série, Universal, 2009.

5. Caprica était par ailleurs un projet pré-existant, soumis au studio Universal par Rémi Aubuchon, avant d'être retravaillée pour intégrer l'hyper-diégèse Galactica ( http:// www.denofgeek.com/tv/battlestar-galactica/20401/ronald-d-moore-interview-capricabattlestar-galactica-virtuality-and-more, consulté le 10/08/2015).

6. «Commercialism, decadence, technology run amok... Remind you of anything? », « Daybreak, Part $1 \& 2 », 1: 33: 00$.

7. La série s'inspire de l'esthétique du film noir popularisée en science-fiction par des films comme Alphaville (Jean-Luc Godard, 1965), Blade Runner (Ridley Scott, 1982) et surtout Gattaca (Andrew Niccol, 1997) : personnages habillés à la façon des années 1950, roulant en Citroën DS, etc. Plus encore que Galactica, la série cherche à s'abstraire d'une esthétique futuriste trop exotique et défamiliarisante, à brouiller un peu plus les repères spatiaux et temporels, et à supprimer les aspects les plus génériques et clivants de la science-fiction traditionnelle.

8. Dave Howe, président de la chaîne, décrivant Caprica, http://www.newsarama.com/1645-sci-figreenlights-caprica-series.html, consulté le 15/08/2015.

9. «It's about a society that's running out of control with a wild-eyed glint in its eye », http:// variety.com/2009/tv/features/caprica-aims-for-broader-demo-1117998604/, consulté le 01/07/2015.

10. François Gabriel Roussel, "Culture des métavers et culture académique. Quelles interactions? », Communications, 33:1, 2015, en ligne (http://communication.revues.org/5227), consulté le 10/08/2015.

11. "No limits, that's the motto", S01E01, 41:00.

12. Film qui s'ouvrait, lui aussi, sur la vision d'une boîte de nuit décadente.

13. Cf. par exemple Gamer (Mark Neveldine, Brian Taylor, 2009), ainsi que Surrogates (Jonathan Mostow, 2009), qui mettent en scène une décadence morale très similaire, et utilisent de même la boîte de nuit décadente comme métonymie de l'anarchie menaçante.

14. Le cataclysme qui ouvre Galactica (le « Jour du Châtiment ", dira un personnage) n'en est alors que l'épilogue. Le dernier épisode de Caprica annonce ce «jugement dernier » par la prophétie «The Day of Reckoning is coming » (« Apotheosis », S01E18, 40:00).

15. « Père, pardonne-leur, car ils ne savent pas ce qu'ils font », Luc 23:34.

16. "A robot, or a person, has two parts: hardware and software. The hardware is the actual physical material involved, and the software is the pattern in which the material is arranged. Your brain is hardware, but the information in the brain is software. The mind [...] memories, habits, opinions, skills [...] is all software ", Rudy Rucker, The Ware Tetralogy: Software, Prime Books, 2010 [1982], édition pdf, p. 111.

17. Julien Offroy de La Mettrie, L'Homme-machine, Paris : Denoël/Gonthier, 1981.

18. « For Case, who'd lived for the bodiless exultation of cyberspace, it was the Fall. [...] The body was meat. Case fell into the prison of his own flesh ", William Gibson, Neuromancer, Londres, HarperCollins, 1995 [1984], p. 12.

19. De la Dignité de l'homme, 1486, http://www.lyber-eclat.net/lyber/mirandola/pictrad.html, consulté le 01/08/2015.

20. Michel Serres, «Le temps humain : de l'évolution créatrice au créateur d'évolution », P. Picq, M. Serres, J-D. Vincent, Qu'est-ce que l'humain?, Paris, Le Pommier, 2008, p. 88.

21. Marvin Minsky, «Will Robots Inherit the Earth? ", Scientific American, octobre 1994, http:// web.media.mit.edu/ minsky/papers/sciam.inherit.html, consulté le 01/08/2015.

22. http://eterni.me/, http://www.livescience.com/37499-immortality-by-2045-conference.html , consultés le 10/08/2015.

23. "On Genes, Memes, Bemes, and Conscious Things", in The Journal of Personal Cyberconsciousness, 1:4, 2006, http://www.terasemjournals.com/PCJournal/PC0104/ 
rothblatt_04a.html ; http://www.parismatch.com/Actu/Environnement/Martine-Rothblatttransgenre-et-transhumaniste-648558, consultés le 10/07/2015

24. «She took a search engine and turned it into a way to cheat death! », S01E01, 59:00. http:// www.bbc.com/future/story/20150122-the-secret-to-immortality, consulté le 13/07/2015. L'épisode de Black Mirror est intitulé « Be Right Back », S02E01 (2013).

25. «A cure for human grief. [...] Who hasn't lost someone that they loved? Who wouldn't do anything possible to bring that loved one back? Well, what if all I had to do was buy the right piece of software that would recreate them? They'd be in the virtual world, yes, but you could visit them every day, talk to them, spend time with them again, say the things that you'd always wished you'd said. Maybe ultimately we can even find a way to get them bodies in the real world. They would be a part of our lives once more. The ultimate drug. To heal the ultimate pain. What do you think that's worth to someone? », « End of Line ", S01E10, 10:00.

26. «Imagine never having to say goodbye to your loved ones again. Imagine a future without loss, brought to you today. 'Grace', by Graystone. Because some memories should live forever ", «False Labor », S01E13, 08:00.

27. Remi Aubuchon, Ronald D. Moore, Caprica, cinquième version, 28 septembre 2006, p. 97.

28. «Who's to say her soul wasn't copied too? », S01E01, 59:53.

29. "You know what your brain is, Joseph? It's a database and a processor, that's all. Information, and a way to use it ", S01E01, 59:15.

30. "The human brain contains roughly $300 \mathrm{~GB}$ of information. Not much when you get right down to it ", S01E01, 44:52. Personne ne sait vraiment quelle quantité d'informations le cerveau humain est capable d'emmagasiner, ni même si une telle analogie informatique est valide, mais les estimations sont en général beaucoup plus importantes.

31. Aubuchon, Moore, Caprica, p. 102.

32. Robert De Niro dans Godsend (Nick Hamm, 2004), DVD, Metropolitan, 28:00.

33. "She's a copy, but... she's a perfect copy ", S01E01, 59:34.

34. Pour reprendre le titre d'une série créée l'année précédente (2009) par Ron Moore (seul le pilote a été produit) et qui s'intéressait également aux rapports entre réel et virtuel.

35. "There's an axiom in my business: A difference that makes no difference is no difference ", S01E01, 59:40. C. Doyle, W. Mieder, F. Shapiro, The Dictionary of Modern Proverbs, New Haven, Yale University Press, 2012, édition kindle, entrée "A difference that makes no difference is no difference $»$.

36. http://hplusmagazine.com/2015/01/15/mindclones-social-media-new-research-stanfordsuggests-feasibility/, consulté le 10/07/2015.

37. Le thème, s'il est très contemporain, n'est pas tout à fait nouveau en science-fiction. Un des romans les plus aboutis sur le sujet est Permutation City (La Cité des Permutants), de Greg Egan, Londres, Orion, 1994.

38. En cela Caprica s'éloigne de la représentation proposée par Black Mirror, qui montrait qu'en définitive il était impossible de recopier tous les éléments qui fondent la personnalité.

39. " A person is much more than just a bunch of usable data », S01E01, 47:00.

40. Gilbert Ryle, The Concept of Mind, Londres, Routledge, 1949. Le terme est devenu très populaire depuis en science-fiction.

41. Gilbert Ryle, The Concept of Mind, 1-14.

42. «I don't feel like a copy ", S01E01, 47:00.

43. «Imagine a world in which death has been conquered. In which eternal life is not just a dream, but a reality. [...] Only they will savor a life everlasting in a virtual heaven that we have built », « Unvanquished », S01E10, 6:30.

44. «We live in a remarkable era. Myth and mystery have been replaced by reason, science. I offer you a religion that removes the need for faith. A religion of certainty that reflects the wonder of all we have created », « Unvanquished », S01E10, 07:25. 
45. « Apotheosis », S01E18; 35:00.

46. « Apotheosis », S01E18, 33:50.

47. Erick Davis, TechGnosis, New York, Harmony Books, 1998, p. 92-195.

48. Transcendence (Wally Pfister, 2014), Warner Bros., DVD, 10:13.

49. Chronologie donnée par un des producteurs exécutifs, Kevin Murphy, dans le commentaire audio de l'épisode.

50. «Well, I think people are smart enough to realize that, as useful as they are, Cylons are simply tools, nothing more, and to forget that, to blur the distinction between man and machine, and to attribute human qualities, is folly ", « Apotheosis ", S01E18, 37:40.

51. http://ieet.org/index.php/IEET/more/3202/, http://hplusmagazine.com/2010/01/19/ caprica-birth-cylons/; http://humanityplus.org/2009/08/woodstockffo9/, consultés le 20/07/2015.

52. http://www.nationalreview.com/human-exceptionalism/325209/surrogates-and-capricasplendid-sci-fi-warnings-against-transhumanism, consulté le 20/07/2015.

53. Je me permets de renvoyer ici à mon propre travail de thèse, L'utopisme technologique dans la science-fiction hollywoodienne, 1982-2010: transhumanisme, posthumanité et le rêve de "l'hommemachine ", 2011, disponible en ligne : https://hal.archives-ouvertes.fr/file/index/docid/779615/ filename/20686_ACHOUCHE_2011_archivage.pdf.

\section{RÉSUMÉS}

Cet article étudie la série de science-fiction Caprica sous l'angle des utopismes technologiques qui y sont représentés. Il examine plus particulièrement la conception mécaniste et dualiste du corps et de la conscience telle que représentée dans la série, qui imagine la naissance d'une intelligence artificielle d'un nouveau type et explore le rêve de l'immortalité digitale. La série souligne ainsi l'importance de l'aspiration spirituelle et du gnosticisme dans les techno-utopismes en général et dans le transhumanisme en particulier, s'éloignant progressivement des termes traditionnels du débat opposant le naturel à l'artificiel ou le matérialisme au spirituel.

This article examines the science-fiction TV show Caprica from the perspective of the technological utopianisms that are represented in the series. It explores the mechanistic and dualistic conception of the body/mind relationship as represented in the series, conveyed through the creation of a new kind of artificial intelligence and the dream of digital immortality through mind uploading or copying. Finally, the show emphasizes how spiritual aspirations related to Gnosticism underpin techno-utopianism as a whole and transhumanism in particular, gradually distancing itself from the traditional debate of natural vs. artificial or materialism vs. spirituality.

\section{INDEX}

Keywords : Caprica, Battlestar Galactica, transhumanism, posthumanity, utopia, dystopia, AI, robot, cyborg, Singularity.

Mots-clés : Caprica, Battlestar Galactica, transhumanisme, posthumanité, utopie, dystopie, IA, robot, cyborg, Singularité 